\title{
ADVANCED 3D TECHNOLOGY IN SUPPORT OF THE BIM PROCESSES IN THE CULTURAL HERITAGE: IN-DEPTH ANALYSIS OF THE CASE STUDY OF THE ROMAN FLUVIAL PORT OF AQUILEIA (ITALY)
}

\author{
S. Pratali Maffei ${ }^{1}$, E. Canevese ${ }^{2}$, T. De Gottardo ${ }^{3}$, L. Pizzol ${ }^{4}$ \\ ${ }^{1}$ Department of Engineering and Architecture, University of Trieste, Italy, pratali@units.it \\ ${ }^{2}$ CEO Virtualgeo, Sacile (PN), Italy, erminio.canevese@virtualgeo.it \\ ${ }^{3}$ R\&D Manager Virtualgeo, Sacile (PN), Italy, tiziano.degottardo@virtualgeo.it \\ ${ }^{4}$ Freelance researcher, Montebelluna (TV), Italy, laurapizzo190@gmail.com
}

KEY WORDS: BIM for Cultural Heritage (HBIM), Advanced 3D, conservation, architectural heritage, Virtualgeo, Aquileia, Roman fluvial port

\begin{abstract}
:
Advanced 3D technology, in line with the directives of the European Commission's Reflective 7 - Horizon 2020 project, allows to represent in an organized and accurate way (geometrically and chromatically speaking) any type of artefact belonging to the Cultural Heritage. This article will deal with the specific case-study of the research carried out in the archaeological site of the Roman fluvial port of Aquileia, in collaboration with the University of Trieste. Thirtysix MA Architecture students attending the experimental course in "Technologies for the conservation and enhancement of architectural heritage" were involved. The Advanced 3D model of the fluvial port was conveniently divided into 15 sectors, in order to allow the assignment of each sector to a different group of students. The students, using the tools provided by the proprietary EasyCUBE PRO software, were able to create a digital database with an accurate 3D representation of all types of degradation affecting the archaeological structures existing in situ. Starting from the digital database, the students created a report containing the surfaces, volumes, count of the specific elements and all the other textual and graphical data related to the degradation processes. The collected data allowed to produce a metric estimate of the restoration intervention and to realize visual simulations of the post-intervention result. This experience gave the chance to evaluate the advantages of creating a digital database of 3D representations of the various forms of degradation, also in terms of possible future developments.
\end{abstract}

\section{INTRODUCTION}

This contribution takes up and deepens the techniques of investigation and study of the forms of degradation, which have already been introduced in a previous paper (Pratali Maffei et al., 2019). This paper focused on the importance of adopting, as a starting point, the Advanced 3D models, exploiting their characteristic of being true to the real (accurate from the geometric and photogrammetric point of view) and structured, in order to be able to extract the metric and photographic information necessary to support the design of conservation interventions, as well as allowing the creation of a precious digital database of the site object of the intervention. The experience has seen the collaboration between Virtualgeo and the University of Trieste and has involved 36 students of the course in "Technologies for the conservation and enhancement of architectural heritage" of the Master in Architecture.

The area considered for the intervention is the archaeological site of the Roman fluvial port of Aquileia (with an extension of about 25,000 square meters) shown in figure 1 . The area has been divided into 15 sectors, each consisting of 3 quadrants as indicated in figure 2 .

Figure 3 shows a graphical representation of sector number 03 where, on the left side, the wireframe of the Advanced 3D model was highlighted. This wireframe was the basis on which, using appropriate software tools that will be described later, the segmentations identifying the various forms of degradation were drawn and calculated.

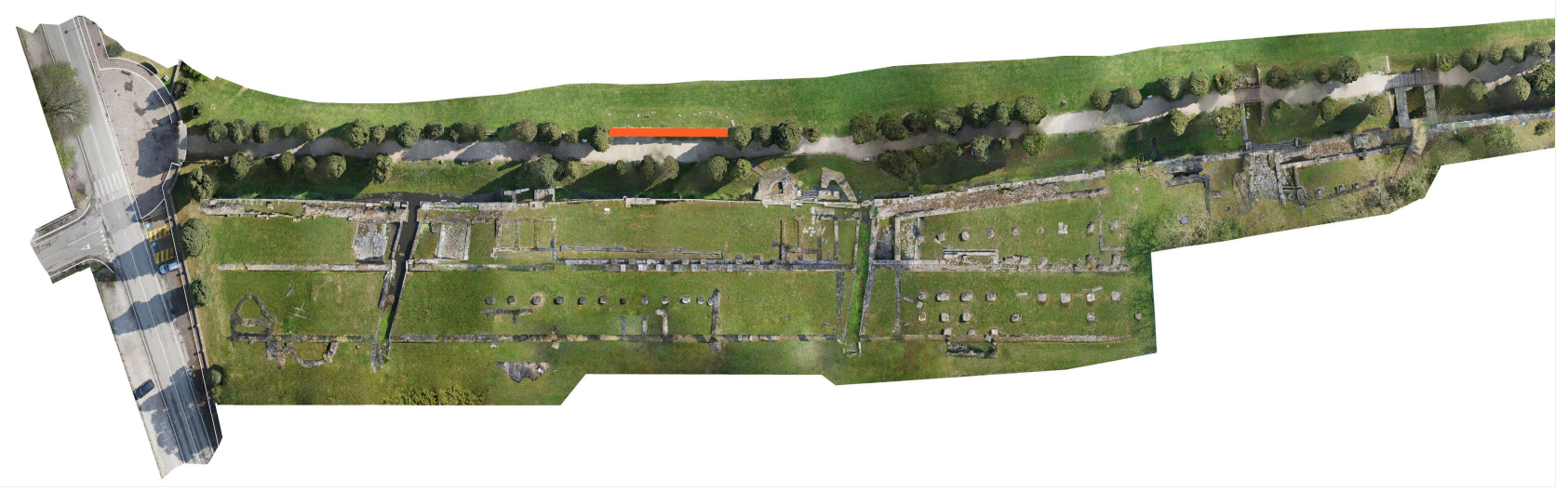

Figure 1. Advanced 3D model of the Roman fluvial port of Aquileia 


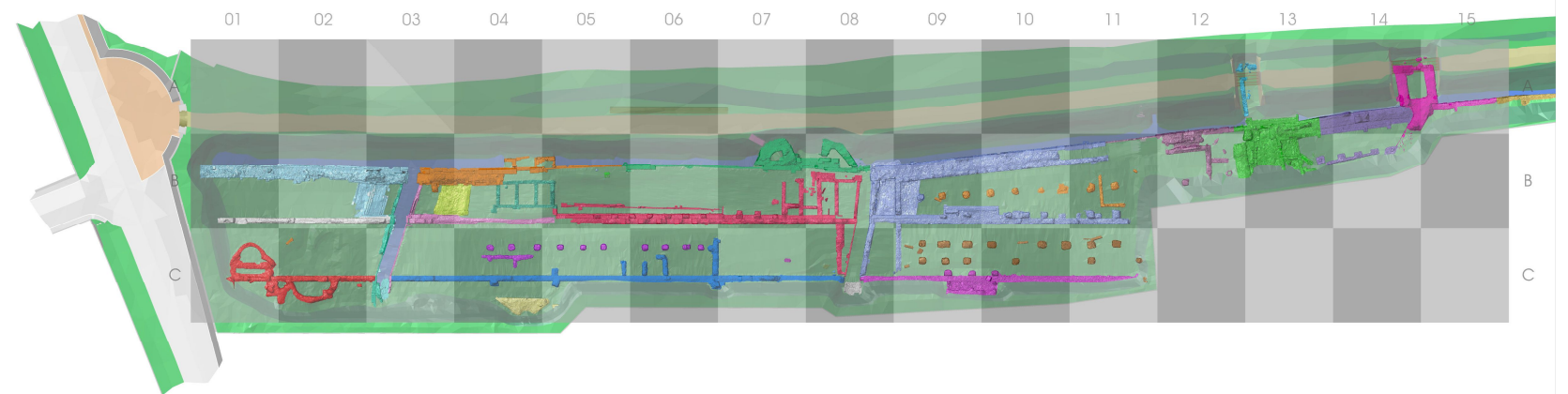

Figure 2. Subdivision of the model in 15 sectors, each consisting of 3 quadrants (A, B, C)

The students were divided into small groups of $2 / 3$ people. Each group was assigned one of the 15 sectors. Within the assigned sector, the students identified and digitally classified all the various forms of degradation. The students also produced post-intervention visual simulations (using photoediting software) and traditional two-dimensional representations (horizontal and vertical sections), suitable for printing on paper and usable on site during the execution of the interventions.

The data produced by each group of students were then collected and combined into a single digital database that allowed us to produce a quantification of the surfaces and a count of the specific elements relating to each type of degradation process present on the site.

After the validation of these data, which were carried out both in didactic terms and in a concrete confrontation with the site manager (Fondazione Aquileia) and the competent control body (Superintendence of Archaeology, Fine Arts and Landscape of Friuli Venezia Giulia), the quantitative data deriving from the segmentations of the degradation areas have

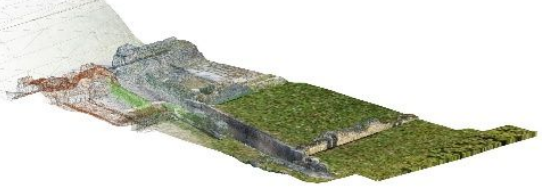

Figure 3. Render of sector number 03 , with highlighted, on the left side, the structure of the $3 \mathrm{D}$ mesh underlying the textures

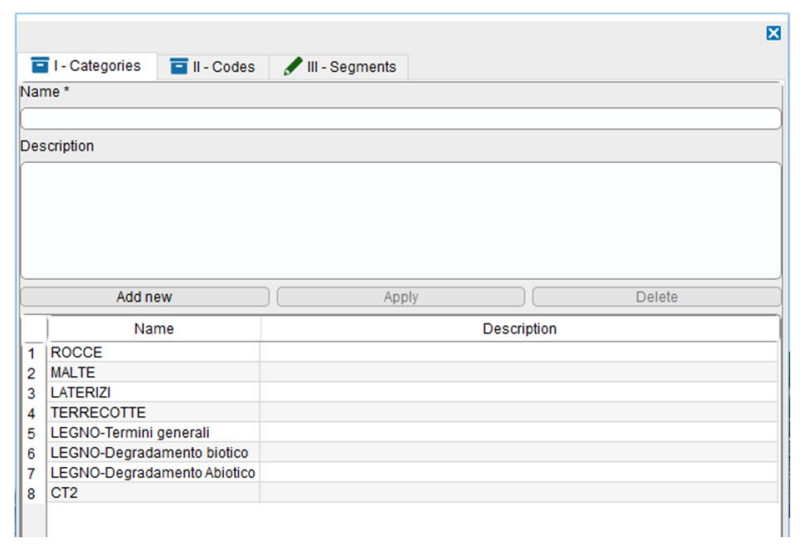

Figure 4. EasyCUBE PRO user interface for inserting/modifying segmentation categories been associated with the relative interventions, so as to obtain an estimative metric calculation.

In this article, in addition to describing in depth how the experience and the tools have been adopted, we will also make some considerations on the data collected and on their potentials.

\section{ANALYSIS AND SEGMENTATION PROCESSES}

As already mentioned in the introduction, each group of students performed the segmentation process on the assigned sector. This process was performed using the EasyCUBE PRO software of the Geomaticscube Ecosystem (Geomaticscube, 2018). Before proceeding to the segmentation of the model a glossary, consisting of a series of categories and codes to describe and classify the various elements to be segmented (materials and degradations), was defined. EasyCUBE PRO allows indeed to import predefined glossaries, to customize them according to the characteristics of the object in question and to store them for subsequent uses. In the specific case of the Aquileia fluvial port, we started from a predefined glossary based on the UNI 11182/2006 standard, which was then appropriately integrated by the students (the integrations were highlighted by a suffix, such as "var. A"), in a homogeneous and coordinated way, in relation to the specific characteristics that were identified during the in situ inspections dedicated to the analysis of the area under study.

The definition of a glossary requires two phases. The first phase is related to the identification of the "categories", the main families of materials in this case, according to which the various types of segmentation will be organized. The introduction of the concept of category is of convenience, as the types of segmentation can be hundreds. Figure 4 shows the interface of the software used for inserting / updating the categories and the list of categories created for this experience (in Italian). The addition of a new category is done simply by specifying the name and an optional description in the appropriate fields.

The second phase consists in defining, for each group previously created, different types of segmentation with homogeneous characteristics, which are called "codes". Figure 5 shows the interface for entering / updating the codes. In the specific case of the image, the codes of the ROCCE (ROCKS) category are reported (in Italian). The insertion of a new code occurs by selecting from the available list the category to which the code will be associated and by specifying the name and an optional description. It is also possible to associate to the segmentations a specific color and two images that identify and describe it.

Once the glossary is defined it can be exported to be used in other projects. 


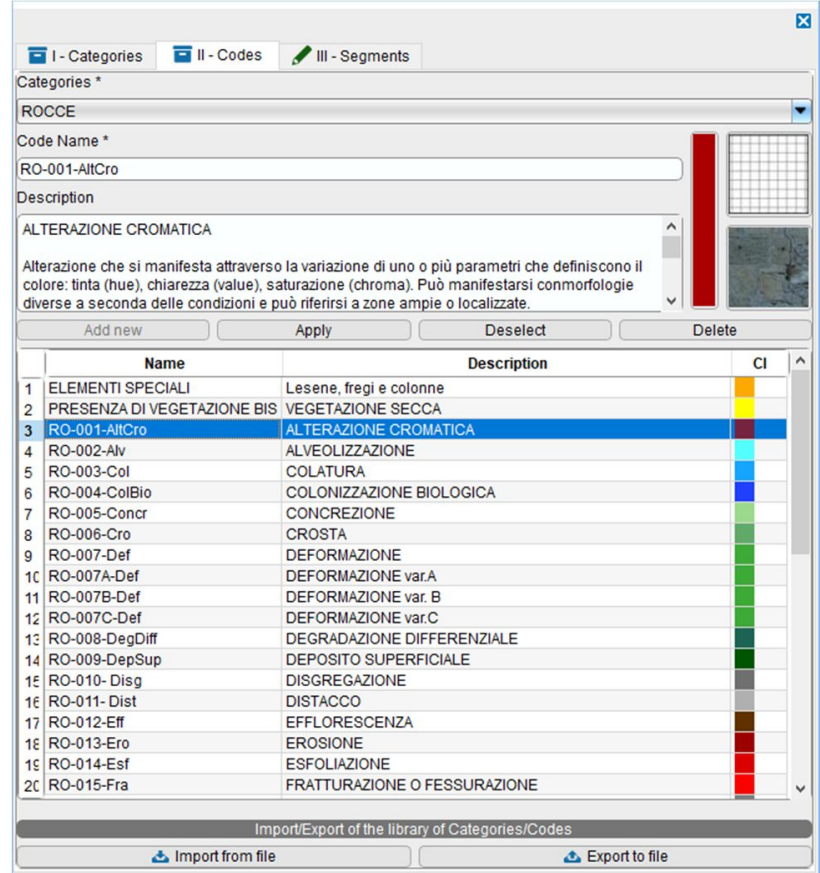

Figure 5. EasyCUBE PRO user interface for inserting/modifying segmentation codes

The next phase was the model segmentation. The students surveyed their sector drawing closed polylines on the 3D model with EasyCUBE PRO tools. This way, they selected 3D surfaces enclosed in the polylines corresponding to specific elements and linked them to a specific code selected from the glossary (see figure 6). Each segmentation can be edited to add or subtract geometry as shown on figure 7 .

In addition to identifying specific elements, areas and volumes affected by particular types of degradation, it was also necessary to signal and quantify the presence or absence of some "objects". This, for example, was the case of the trees to be removed because potentially harmful or of the missing metal clamps that originally linked some stone elements. In these cases, we first defined a code for each type of object and then segmented them, or the areas lacking them, using the relative codes. At the end of the segmentation process, the specific elements were simply counted.

\section{DATA COLLECTION}

During the teaching experience and after the inspections, the need to calculate the volume of some objects also emerged. This is because some elements can not be restored in situ but must be transported to specialized laboratories. In this case the estimate of the cost of the intervention is also linked to the volume of the object itself, and not only to the surface to be treated.

It has therefore been implemented a software tool, called "Working Box", which allows to define the minimum rectangular parallelepiped (box) able to enclose a 3D object. The Working Box is created by specifying 3 points (Figure 8) close to the object, one for the origin of the box and two for the "X" and "Y" Cartesian axes. Subsequently, thanks to specific features of the tool, the sides of the box can be adapted to exactly include the object under examination. Once a box has been defined and adapted as necessary, its geometric properties (i.e. the size of the sides, the area and the volume) are reported at the top of the interface.

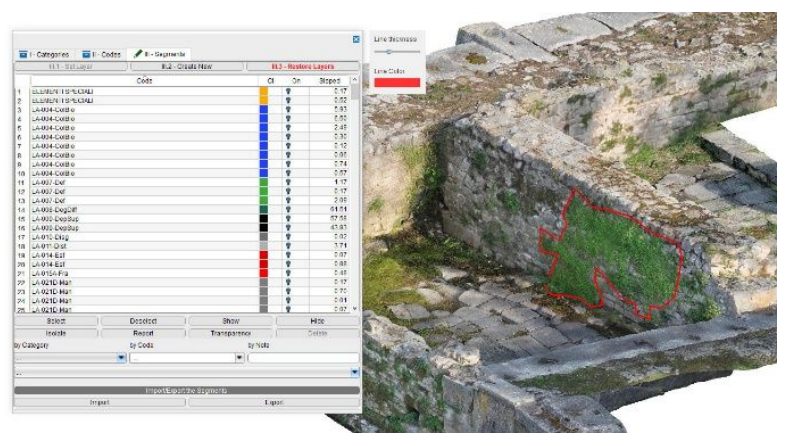

Figure 6. Design phase in EasyCUBE PRO of a closed polyline that identifies the $3 \mathrm{D}$ segmentation area
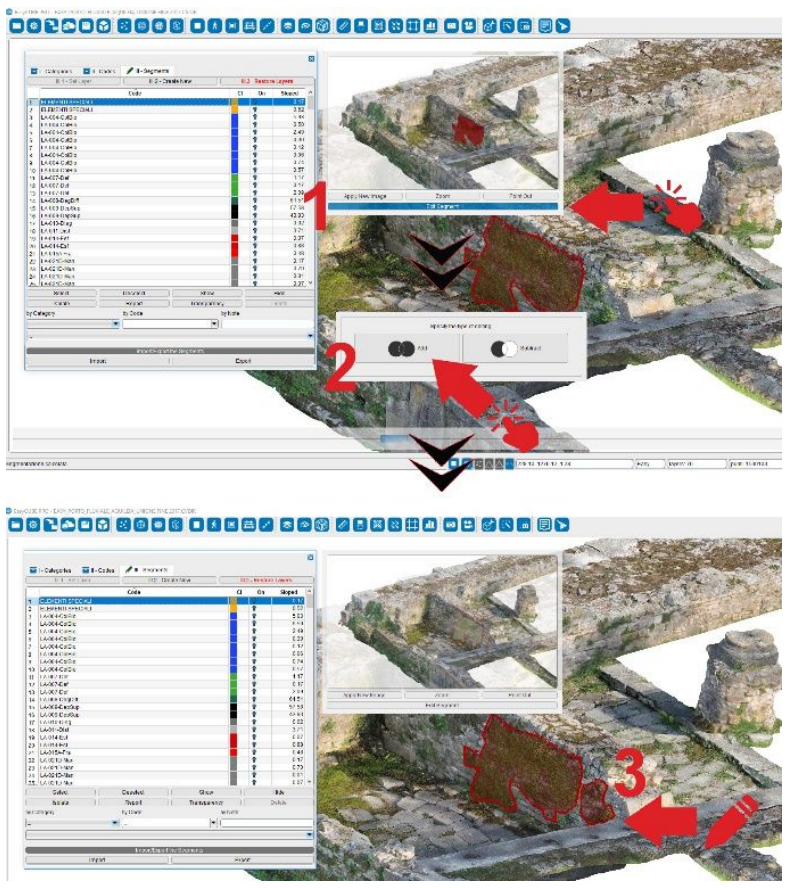

Figure 7. Description of the editing flow of a segmentation in EasyCUBE PRO, in this case with the addition of a portion

The Working Box tool can also be used to display only the area of the 3D model included in the current box, thus facilitating segmentation or analysis operations.

Moreover, by clicking on the "Front", "Back", "Left", "Right", "Top" and "Bottom" keys of the interface in figure 8, the point of view of the corresponding side of the current box is automatically set. This is a very useful feature supporting the display of the images of the corresponding exploded box, as illustrated by the example in figure 9 .

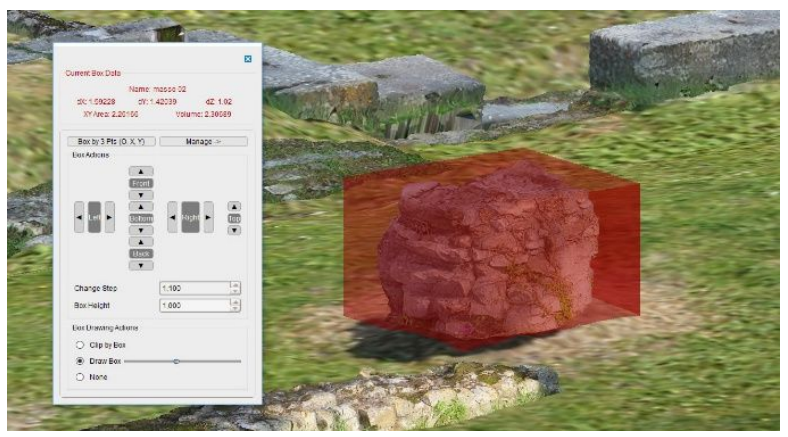

Figure 8. EasyCUBE PRO interface for creating and managing the current working box 


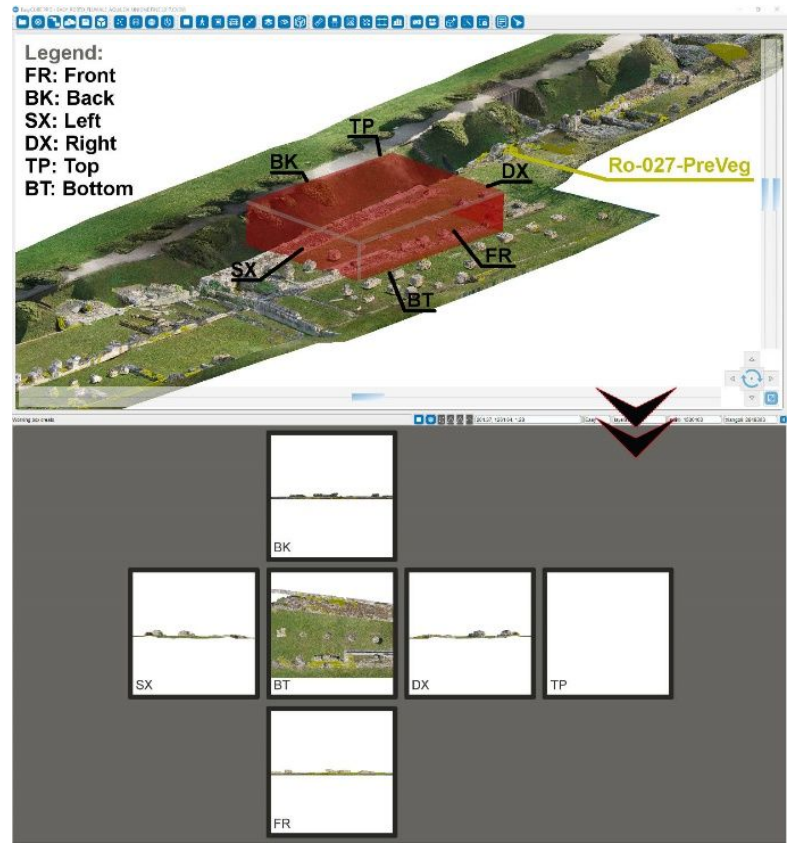

Figure 9. Example of a "exploded box" graphic representation of the degradation with code Ro-027-PreVeg, obtained using the "Working Box" tool

Finally, there is an important feature of EasyCUBE PRO to support the segmentation processes. This feature permits to automatically generate a PDF report (accompanied by a homonymous Excel report containing only the computational data) that groups and summarizes all the information (segmentation positions, graphical representations and textual notes) and computational (partial and total segmentation surfaces) data collected during the segmentation process. Figure 10 shows a portion of the report that illustrates informative data, while figure 11 shows a portion with computational data.

\section{DATA PROCESSING AND EVALUATION OF RESULTS}

All the data obtained by each group through the segmentation process were later assembled in a single proprietary file, thus unifying the database. This file describes the whole site segmented with 46 degradation codes organized in 8 categories.

A global report, counting 335 pages and containing all the information and computational data related to the surveyed degradation processes, was than generated in pdf format.

Figure 12 shows a render of the Advanced 3D model with all the segmentations highlighted.

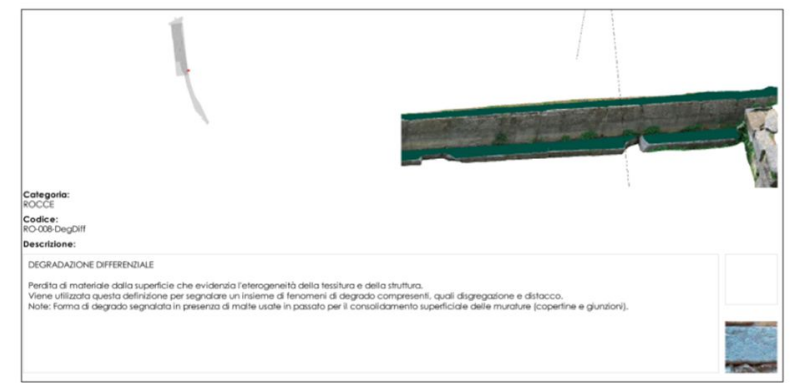

Figure 10. Portion of a PDF report automatically generated by EasyCUBE PRO (informative data)

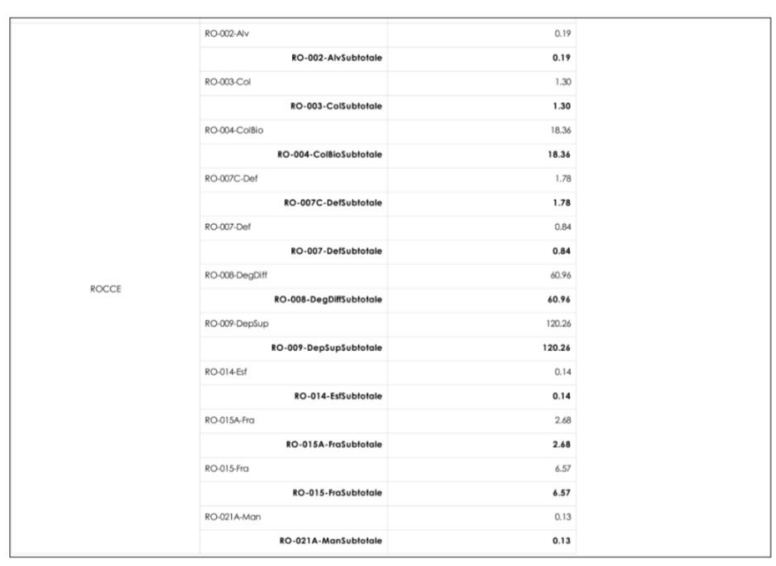

Figure 11. Portion of a PDF report automatically generated by EasyCUBE PRO (computational data)

The final verification of the results obtained was carried out by collating and comparing all the products produced. In the case of apparently anomalous data, a punctual check was carried out, both on the model and on the field, in such a way as to reduce errors and inaccuracies resulting from subjective interpretation, by the 36 students involved in the experience, of reading materials and forms of degradation present.

These cases were very limited (in the order of 5\%) and all justified by situations of doubtful or difficult interpretation, that is from elements where more materials were present or which saw more forms of degradation overlapping.

Once the data were validated, the 15 sectors in which the model was divided were reassembled, so as to obtain a single reference system. Likewise, the various estimating metric calculations have been reunited into a single spreadsheet, which allows both the comparison between the various sectors and the overall quantification of the intervention costs.

In this way, the possibility of including separate phases of intervention was maintained, in relation to the available resources, both by subdividing it by areas and by type of works. Which in fact happened with the realization of the ordinary maintenance works, relating to the removal of incoherent deposits and the elimination of infesting vegetation. The model and the estimative metric calculation can easily be updated by eliminating the segmentations and the quantifications related to these forms of degradation/ intervention, so as to have a discounted representation and cost. It is also evident that the model, at a distance of time, should be reviewed, at least as regards the textures, or the representation of the surfaces (provided that changes in the morphology of the architectural elements have occurred).

In this case it may be sufficient to carry out a new georeferenced photographic campaign "dressing" the geometric model again, and then verify and update the previous segmentations.

\section{CONCLUSIONS}

The use of Advanced 3D models and cutting-edge technological tools in the field of architectural and archaeological restoration offers the opportunity for a significant renewal and enhancement of the current operating procedures.

From a short-term perspective, for example, the implementation of a simple function for the differentiated storage and comparison of the segmentations obtained by various segmentation campaigns (appropriately scheduled) could allow the development of a "historical" and dynamic 


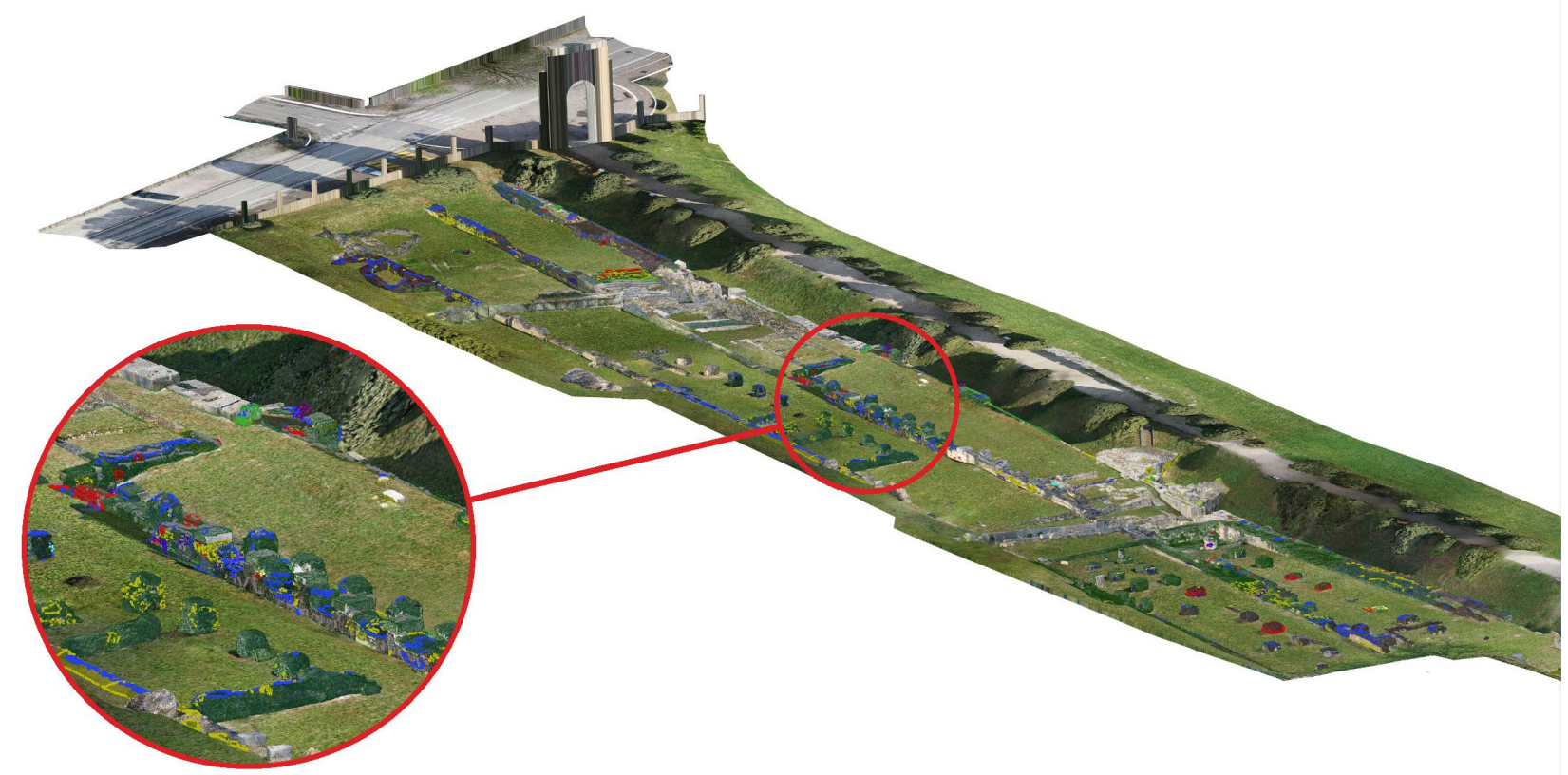

Figure 12. Render of all the segmentations of the various forms of degradation produced by the 36 students

database, which could be used to monitor the evolution of degradation processes. This monitoring could be significant both for a better planning of the restoration interventions (which would thus be based on a more substantial amount of data) and for a more objective evaluation of the effectiveness of the interventions already carried out. In this sense, a comparison of the segmentations related to areas affected by the same degradation processes but treated differently could represent a valid tool to support the identification of the most appropriate solutions for the specific context.

Such a database, although of undoubted use, would still continue to be characterized by a significant limitation: it would be fundamentally static. The various segmentations would in fact continue to be carried out, even after several years, on the same Advanced 3D model, thus not taking into account the morphological evolution of the structures that, especially after restorations, could prove to be significant. To make the model and the connected database more "dynamic", it might be opportune to develop new acquisition and modelling processes to make the implementation of new Advanced 3D models less onerous, especially from an economic point of view.

Another option is the integration of the Advanced 3D model or its data (for example the PDF reports of the segmentation or degradation analysis process) with the simplified BIM model of the subject of the intervention, otherwise defined HBIM (Canevese et al., 2017). This would be beneficial to support both decision-making processes in the design of restoration activities and the cooperation of various professional profiles through the tried and tested BIM tools.

In the medium term, the development of the Advanced 3D technology and the proprietary software of the EasyCUBE family could instead be directed towards new hardware. In particular, the creation of specific applications for wearable devices, such as viewers for mixed reality like Hololens (Coppens A., 2017), could make the abovementioned information immediately available and usable to operators on site. For instance, during a survey or a restoration work, a restorer equipped with a viewer could recall with simple gestures information on the distribution of specific degradation processes, their evolution or, more simply, on the characteristics of the substrate on which he is going to operate.

Even in this sense, collaboration between companies and universities is once again essential, as this experience shows, in order to improve the overall results of research in the field of cultural heritage.

In the present case, this dialogue allowed students to learn and experiment on the ground the use of innovative software, to the developer to implement and improve their functionalities, to the operator of the archaeological site to obtain, without costs, a scientifically based analysis of the state of preservation of the site, the identification of the necessary maintenance and restoration interventions, with the prediction of the relative costs.

\section{REFERENCES}

Canevese E.P., De Gottardo T., 2017. Beyond Point Clouds and Virtual Reality. Innovative Methods and Technologies for the Protection and Promotion of Cultural Heritage, May 2017, https://www.int-arch-photogramm-remote-sens-spatial-infsci.net/XLII-5-W1/685/2017/

Coppens A., 2017. Merging real and virtual worlds: An analysis of the state of the art and practical evaluation of Microsoft Hololens, June 2017,

https://www.researchgate.net/publication/317932970 Mergin $\mathrm{g}$ real and virtual_worlds An analysis of the state of the _art_and_practical_evaluation_of_Microsoft_Hololens

Geomaticscube, 2018. https://www.geomaticscube.com/

Pratali Maffei S., Canevese E., De Gottardo T., 2019. The real in the virtual. The 3D model in the Cultural Heritage sector: the tip of the iceberg, January 2019 , https://doi.org/10.5194/isprs-archives-XLII-2-W9-615-2019 